\title{
In vitro antioxidant capacity of tomato products: Relationships with their lycopene, phytoene, phytofluene and alpha-tocopherol contents, evaluation of interactions and correlation with reflectance measurements
}

\author{
Carla M. Stinco, Francisco J. Heredia, Isabel M. Vicario, Antonio J. Meléndez-Martínez* \\ Food Colour \& Quality Lab, Dept. Nutrition \& Food Science, Universidad de Sevilla, Facultad de Farmacia, 41012 Sevilla, Spain
}

Keywords:

$\alpha$-tocopherol

Carotenoids

Lipophilic antioxidants

Synergistic effect

Reflectance

\begin{abstract}
A B S T R A C T
Tomato products were analysed for their carotenoids and $\alpha$-tocopherol contents to study the contribution of individual compounds to the antioxidant capacity and assess the existence of interactions. Besides, the applicability of reflectance measurements for the estimation of the Trolox Equivalent Antioxidant Capacity (TEAC) was explored. Statistically significant regression coefficients were found between TEAC values and carotenoids and $\alpha$-tocopherol levels. Lycopene seemed to have the highest contribution, followed by phytofluene. The results showed that the antioxidant capacity of phytofluene warrants further investigation. It was observed that there can be interactions between lycopene and $\alpha$ tocopherol, although just the lycopene/ $\alpha$-tocopherol ratio cannot be used to predict the kind of interaction. More knowledge of these interactions would supply new tools for the industry to develop more optimized ingredients. Finally, the lycopene levels and TEAC values can be estimated by considering reflectance readings at certain wavelengths, which is important for field and quality control applications.
\end{abstract}

\section{Introduction}

Lycopene (LYC) is a major carotenoid in tomato and tomatobased product, these products contain other carotenoids (phytoene and phytofluene in the main and much lower levels of others) (Meléndez-Martínez, Fraser, \& Bramley, 2010) and compounds (Adalid, Roselló, \& Nuez, 2010; Borguini \& Ferraz Da Silva Torres, 2009; Erba et al., 2013), which may be related to the diverse health benefits these products are thought to provide (Kun, Ssonko Lule, \& Xiao-Lin, 2006).

Epidemiological studies have associated the consumption of tomato products with a reduced risk of developing diseases like cardiovascular diseases and certain types of cancer, especially prostate cancer (Giovannucci, 2005), although causality is difficult to be established. These health benefits have been frequently

Abbreviations: LYC, lycopene; PT, phytoene; PF, phytofluene; ATOC, $\alpha$-tocopherol; AC, antioxidant capacity; TEAC, Trolox Equivalent Antioxidant Capacity Assay; ThAC, theoretical antioxidant capacity value; ExAC, experimental antioxidant capacity value; SE, synergistic effects.

* Corresponding author.

E-mail address: ajmelendez@us.es (A.J. Meléndez-Martínez). attributed to the presence of antioxidants. However, many aspects related to their possible mode of action remain largely obscure.

Due to the dietary and economic importance of tomatoes (Thakur, Singh, \& Nelson, 1996) as well as the health-promoting actions attributed to carotenoids and other compounds, the study of their contents in tomato products with different goals remains a very important research topic (Adalid et al., 2010; Heredia, Peinado, Barrera, \& Grau, 2009; Tedeschi et al., 2011). Although the theoretical and in vitro AC of some carotenoids is unarguable, their role as antioxidants in vivo and the underlying mechanistic aspects still raise controversy. In any case, the evaluation of the scavenging capacity of extracts can be useful to assess their usefulness as food ingredients or to gain insight into how different antioxidants interact in the presence of certain electrophiles.

Carotenoids possess a characteristic electron-rich chromophore, which is involved in the AC of these compounds towards different species (Galano \& Francisco-Marquez, 2009; Martínez, Stinco, \& Meléndez-Martínez, 2014). LYC as an antioxidant has been associated to the possible health benefits derived from the consumption of tomato. In contrast, little attention has been paid to the colourless carotenoids phytoene (PT) and phytofluene (PF), which are also found at high levels in tomato products. They are efficiently 
absorbed and accumulated by humans and other mammals (Meléndez-Martínez, Stinco, Liu, \& Wang, 2013) and have been found to behave like antioxidants both by theoretical and experimental approaches (Martínez et al., 2014). In this regard, some studies on animals suggest a preferential accumulation of PT and PF over LYC in some tissues, which indicates that the absorption and/ or metabolism of these compounds may be different from that of LYC (Meléndez-Martínez et al., 2013b). In fact, PT and PF may be bioactive and be related to some of the biological effects attributed to LYC on its own, as tomatoes or tomato carotenoid extracts rather than pure LYC are commonly used in the studies (MeléndezMartínez, Mapelli-Brahm, Benítez-González, \& Stinco, 2015).

There is a wealth of studies devoted to the assessment of the AC of individual carotenoid or carotenoid extracts, although the evaluation of the possible existence of interactions between antioxidant species, with few exceptions (Liu, Shi, Colina Ibarra, Kakuda, \& Jun Xue, 2008; Shi et al., 2007; Shixian et al., 2005; Zanfini, Corbini, La Rosa, \& Dreassi, 2010), has been largely overlooked. This information is of little value if it comes to try to extrapolate the observations of in vitro studies in solutions to an in vivo scenario. However, it is interesting for the food industry as different compounds with AC can be used as food ingredients (carotenoids, ATOC, etc.), which may interact to cause synergistic or antagonistic effects towards the protection against oxidants.

In this study, we have assessed the levels of LYC, PT, PF and ATOC and the AC of lipophilic extracts from diverse tomato genotypes and products with four main purposes: 1) to evaluate the differences in their Trolox Equivalent Antioxidant Capacity (TEAC), 2) to estimate the relative contribution of individual antioxidants to the TEAC, 3) to evaluate the possible existence of interactions between carotenoids and tocopherols in the scavenging of the ABTS+ and 4) to assess the usefulness of reflectance measurements for the rapid and non-destructive estimation of this activity. This information can be interesting for the industry in order to optimize the simultaneous use of carotenoids and tocopherols as versatile food ingredients that provide both technological and health benefits. Furthermore, it can be used for the rapid evaluation of LYC and AC in field and quality control applications.

\section{Materials and methods}

\subsection{Chemicals}

Analytical grade methanol, acetone and trichloromethane for extractions were purchased from Carlo-Erba (Milan, Italy). HPLCgrade methanol and methyl-tert-butyl-ether (MTBE) for the chromatographic analyses were procured from Merck (Darmstadt, Germany). The ATOC standard was purchased from Sigma-Aldrich (Taufkirchen, Germany). The carotenoid standards (LYC, PT and PF) were isolated from appropriate sources according to recommended procedures routinely used in our laboratory. ABTS, 2,2'-azinobis (3ethylbenzothiazoline-6-sulfonic acid) diammonium salt, and potassium persulfate (di-potassium peroxdisulfate) were purchased from Sigma-Aldrich (Taufkirchen, Germany).

\subsection{Samples}

Two different groups of tomatoes were considered. The first group consisted of 22 samples of tomato fruits from different commercial types (Cherry, Salad, Raf, Kumato, Rambo, Bola, Velazco, Canario, Daniella, Pear, and Cluster). The second group included 22 processed tomato products of different brand were purchased from a local supermarket. The commercial samples within this group were subjected to some type of industrial processing, like mechanical processes (chopping, peeling, crushing, etc.) and/or thermal treatments like concentration. Moreover, most of these products were subjected to sterilization.

\subsection{Sample preparation}

The peel, jelly and seeds of the fresh tomatoes were removed and discarded. All the samples were homogenized by using a mechanical blender and their reflectance spectra were subsequently obtained by spectroradiometry measurements. Afterwards, the tomato homogenates were freeze-dried for $48 \mathrm{~h}$ and stored at $-20{ }^{\circ} \mathrm{C}$ until the HPLC analyses.

\subsection{Reflectance measurements}

The reflectance spectra were obtained by means of a CAS $140 \mathrm{~B}$ spectroradiometer (Instrument Systems, Germany) fitted with a Top 100 telescope optical probe, a Tamron zoom mod. SP 23A (Tamron USA, Inc., Commack, NY), and a white light $150 \mathrm{~W}$-metalhalide lamp Phillips MHN-TD Pro (12,900 lumen, $4200 \mathrm{~K}$ colour temperature) as external source of illumination. Blank measurements were made with distilled water, against a white background. The spectroradiometer was set to take three consecutive measurements of each sample, and the entire visible spectrum $(380-770 \mathrm{~nm})$ was recorded with a bandwidth of $2 \mathrm{~nm}$.

\subsection{Analysis of carotenoids and tocopherols}

\subsubsection{Isoprenoids extraction}

The analysis of these isoprenoid compounds was performed as described in Stinco et al. (2013).

\subsubsection{HPLC analysis, identification and quantitative analysis of carotenoids and $\alpha$-tocopherol}

The HPLC conditions and criteria for the identification of geometrical isomers are explained in detail (Stinco et al., 2013). The levels of LYC, PT, PF and ATOC were obtained from dose-response calibration curves. Due to the lack of appropriate standards of $(Z)-$ isomers of carotenoids and data on their extinction coefficients all the isomers were quantified with the calibration curve made with the (all-E)-LYC standard.

\subsection{Evaluation of the in vitro AC of tomato lipophilic extracts}

The assessment of the in vitro AC of lipophilic extracts was carried out according to the ABTS radical cation decolourisation assay (Re et al., 1999). The measurements were carried out using aliquots $(20-100 \mu \mathrm{L})$ of the same extracts used to determine isoprenoids by HPLC. The 2,2'-azinobis(3-ethylbenzothiazoline-6sulfonic acid) radical cation $\left(\mathrm{ABTS}^{+}\right)$was produced by reacting an aqueous solution of ABTS ( $7 \mathrm{mM}$ ) with potassium persulfate (2.45 mM, final concentration) and allowing the mixture to stand in the dark at room temperature for 12-16 h. Trolox (6-hydroxy2,5,7,8-tetramethylchroman-2-carboxylic acid; Sigma) was used as a standard. One $\mathrm{mL}$ of the ABTS radical solution was added to the cuvette and the absorbance was measured at time 0 . Subsequently, $5 \mu \mathrm{L}$ and $15 \mu \mathrm{L}$ of the extracts were added to the cuvette. It stirred and incubated at $30^{\circ} \mathrm{C}$. After $6 \mathrm{~min}$, the absorbance was measured at $734 \mathrm{~nm}$ on a HP-8453 spectrophotometer equipped with temperature controller. The dose-response curve for Trolox consisted of plotting the absorbance at $734 \mathrm{~nm}$ as a percentage of the absorbance of the uninhibited radical cation (blank) and was based on triplicate determinations. The AC of the lipophilic extracts was expressed as millimol of Trolox equivalents per kg of dry weight. 
2.7. Single antioxidant solutions and pre-defined mixtures of lycopene and $\alpha$-tocopherol

LYC, ATOC, PT and PF (different concentrations) were assayed individually using the ABTS assay as previously described. Mixtures of LYC and ATOC were prepared and analysed by the same method. To determine possible synergistic effects (SE), a theoretical antioxidant capacity value (ThAC) was calculated and compared with the experimental antioxidant capacity value (ExAC) for each mixture as proposed by Fuhrman, Volkova, Rosenblat, and Aviram (2000). ThAC was calculated as the sum of the antioxidant capacities of the individual components of the mixture. Whenever the ExAC values were greater than the ThAC values (that is, when ExAC/ ThASC > 1) it was considered that synergistic interactions could have taken place.

\subsection{Statistical analysis}

The results are expressed as mean and standard deviation of three independent determinations. Appropriate statistical models were applied to evaluate the existence of significant differences and correlations and to select variables. Specifically, one-way analysis of variance (ANOVA) followed by Tukey's test and Stepwise Linear Discriminant Analysis (SLDA) were carried out. Correlations between carotenoids or ATOC contents and TEAC values were evaluated by both simple and multiple regressions. In case of multiple regressions, Partial Least Squares Regression (PLSR) was used. It is a procedure used to relate a large number of independent variables (i.e.: spectrum) to the prediction of one or few response variables (chemical concentration). Performance of the prediction models were evaluated using the coefficient of determination in calibration $\left(R^{2}\right)$ and coefficient of determination in cross-validation $\left(R_{c v}^{2}\right)$. All statistical analyses were performed with the Statistica V.8.0 software.

\section{Results and discussion}

\subsection{Carotenoids and $\alpha$-tocopherol levels}

Table 1a and 1b (supplementary material) summarize the LYC, PT, PF and ATOC contents as well as the AC of lipophilic extracts of the samples.

As expected, the mean levels of all the LYC isomers were significantly $(p<0.05)$ higher in processed than in fresh tomatoes. Five (Z)-isomers, namely (5Z)-LYC, (9Z)-LYC, (5Z, 9'Z)-LYC, (13Z)-LYC and (15Z)-LYC, were tentatively identified on the basis of previously reported data (Fröhlich, Conrad, Schmid, Breithaupt, \& Böhm, 2007; Meléndez-Martínez et al., 2013b; Stinco et al., 2013). The major (Z)isomer in both types of samples was (5Z)-LYC. The differentiation between different LYC isomers is important as they are thought to have different properties, bioavailabilities and metabolic fates (Guo, Tu, \& Hu, 2008; Honest, Zhang, \& Zhang, 2011). In fact, it is well known that human plasma and tissues contain high levels of $(Z)$ isomers of LYC, despite the fact most of the dietary LYC is (all-E)-LYC (Fröhlich, Kaufmann, Bitsch, \& Böhm, 2006; Stahl \& Sies, 1992). Most tomato genotypes also contain high levels of the colourless carotenes PT and PF (Meléndez-Martínez et al., 2010; Raffo et al., 2002). The LYC to PT ratio ranged from 2.8 -fold to 16.6 -fold in fresh tomatoes and from 4.8 -fold to 11.4 -fold in processed tomatoes. In the case of the LYC to PF ratio, the values ranged from 9.9-fold to 36.8-fold in fresh tomatoes and from 9.2-fold to 32.1fold in processed tomatoes.

In this study, the average concentrations of PT and PF were lower (by 61 and 71\%, respectively) in fresh tomatoes with respect to processed tomatoes.
Tomatoes are also good sources of other antioxidant isoprenoids, like ATOC. As observed for carotenoids, processed tomatoes showed higher levels of ATOC. Specifically, the mean ATOC content of the different tomato fruits analysed in this study was $320 \pm 103 \mathrm{mg} / \mathrm{kg} \mathrm{DW}$, whilst that for processed foods was $1075 \pm 288 \mathrm{mg} / \mathrm{kg}$ DW, that is, almost 3.5-fold higher.

The differences in the levels of secondary metabolites in plants (and therefore in their derived products) are owed to factors like genotype, maturity stage, climate and agronomic conditions, etc. In the case of tomato carotenoids, this subject has been addressed in many studies (Abushita, Daood, \& Biacs, 2000; Raffo et al., 2002). Concerning processed tomatoes, additional technological factors like mechanical homogenizations and thermal treatments can also affect the secondary metabolites contents (Dewanto, Wu, Adom, \& Liu, 2002).

\subsection{In vitro AC of lipophilic tomato extracts. Assessment of} relationships with carotenoids and $\alpha$-tocopherol contents

The results of the analyses are shown in electronic supplementary material Table $1 \mathrm{a}$ and $1 \mathrm{~b}$ The mean TEAC values for fresh and processed tomatoes were $3.81 \pm 1.59$ and $8.96 \pm 2.65 \mathrm{mM}$ Trolox equivalents/kg DW, respectively.

As expected, the analysis of variance (ANOVA) revealed that, as observed for the LYC and ATOC levels, there were significant differences $(p<0.001)$ in the TEAC values between fresh and processed tomato products.

To assess correlations between TEAC values and the levels of antioxidants, simple linear regression analyses were carried out. Good correlations between the TEAC values and the LYC $(r=0.93)$ and ATOC $(r=0.84)$ levels were found when all the samples were considered altogether. Both correlations were significant at $p<0.05$. When the samples were considered as two independent groups (fresh and processed), the values of the regression coefficients obtained were lower, above all in the case of ATOC, albeit still significant (Table 1). These data are in agreement with the observations made by Zanfini et al. (2010) and Raffo et al. (2002), who reported high positive correlation between LYC and the TEAC values ( $\mathrm{r}=0.96$ in both cases) of tomato extracts. As it can be observed in Table 1, significant and high simple regression coefficients were found between the TEAC values and the levels of PT and $P F$, which seemed to suggest that these carotenes may also exhibit scavenging capacity towards ABTS ${ }^{+}$. Similarly, Raffo et al. (2002) also found very good correlations between the PT $(r=0.94)$ and $\mathrm{PF}(\mathrm{r}=0.89)$ contents and the AC of cherry tomato extracts.

The TEAC values of individual compounds, determined as the decreases in the absorbance (inhibition) of the $\mathrm{ABTS}^{+}$solution as a

Table 1

Correlation coefficients ( $r$ ) between antioxidants levels and TEAC values. Significant correlations $(p<0.05)$ are denoted in italics.

\begin{tabular}{|c|c|c|c|}
\hline \multirow[t]{2}{*}{ Compounds } & All samples & Fresh & Processed \\
\hline & $\mathrm{n}=44$ & $\mathrm{n}=22$ & $\mathrm{n}=22$ \\
\hline ATOC & 0.835 & 0.448 & 0.552 \\
\hline PT & 0.886 & 0.748 & 0.705 \\
\hline $\mathrm{PF}$ & 0.870 & 0.698 & 0.687 \\
\hline (15Z)-LYC & 0.524 & 0.815 & 0.383 \\
\hline (13Z)-LYC & 0.922 & 0.856 & 0.793 \\
\hline (9Z)-LYC & 0.834 & 0.583 & 0.600 \\
\hline (All-E)-LYC & 0.923 & 0.826 & 0.807 \\
\hline (5Z)-LYC & 0.829 & 0.911 & 0.486 \\
\hline$\sum$ Z-isomers & 0.888 & 0.912 & 0.649 \\
\hline$\sum \mathrm{LYC}$ & 0.931 & 0.845 & 0.820 \\
\hline
\end{tabular}

Abbreviations: LYC, lycopene: PT, phytoene; PF, phytofluene; ATOC, $\alpha$-tocopherol 
function of the amount of antioxidant added, were $3.5 \mathrm{mM}$ Trolox equivalents (for LYC) and 2.0, 1.0 and 2.6 mM Trolox equivalents, for ATOC, PT and PF, respectively (Martínez et al., 2014). The TEAC values obtained for lycopene and ATOC were slightly higher than those previously reported (Miller, Sampson, Candeias, Bramley, \& Rice-Evans, 1996; Zanfini et al., 2010). Unlike LYC and ATOC, the in vitro AC of the colourless carotenoids PT and PF has not been assessed in traditional works on the AC of carotenoids (Miller et al., 1996; Re et al., 1999; Woodall, Lee, Weesie, Jackson, \& Britton, 1997). The results reported here indicate that they exhibit scavenging capacity towards ABTS ${ }^{+}$, above all PF, whose TEAC was even higher than that of ATOC. These results were somewhat unexpected as the chromophores of these carotenoids are considerably shorter (PT has 3 conjugated double bonds and PF five) than those of carotenoids traditionally regarded as good in vitro antioxidants (like, LYC or $\beta$-carotene, among others) with 9 or more conjugated double bonds. The results of a previous study (Martínez et al., 2014), the information inferred from the regression models mentioned above (Tables 2 and 3 ) and the assessment of the TEAC invites to further evaluate the AC of PT and PF in other matrices and towards other oxidants.

The relative contribution of lycopene isomers, PT, PF and ATOC to the TEAC was assessed by multiple regression. The corresponding coefficients of determination in calibration $\left(R^{2}\right)$ and crossvalidation $\left(R^{2}{ }_{c v}\right)$ are shown in Table 2 . All the $R^{2}{ }_{c}$ values were $>0.87$ and the best prediction model of the AC was obtained when all the major tomato carotenoids (LYC, PT and PF) and ATOC were considered $\left(R^{2}{ }_{c}=0.90\right)$. Coefficients of determination in crossvalidation $\left(\mathrm{R}_{\mathrm{cv}}^{2}\right)$ were higher than 0.84 in all models. To obtain some information about the possible contribution of each independent variable to the prediction of $A C$, the standardized regression coefficients $(\beta)$ were taken into account. In this sense, the carotenoids with higher weights were LYC and PF.

\subsection{AC of pre-defined mixtures of lycopene and $\alpha$-tocopherol}

Although the TEAC of individual carotenoids and lipophilic extracts have been the subject of many studies, very few address the possible existence of interactions between different antioxidants. This information can be interesting for the formulation of foods with enhanced oxidative stability. Besides it could be important in economic terms for the food ingredient industry as the existence of synergistic interactions could be harnessed to reduce the amount of antioxidants added to the products.

To ascertain possible synergistic effects (SE), both the experimental and the theoretical TEAC of different mixtures of LYC (used in the industry as a colourant and functional ingredient) and ATOC (used in the industry as an antioxidant) were evaluated. The results are summarized in Table 3.

Of the 35 mixtures tested, there were seven (M4, M5, M14, M15,
M18, M19 and M16) with SE > 1, although only in three cases statistically significant effects were observed (M5, M15 and M26). Contrastingly, there were 18 mixtures with statistically significant $\mathrm{SE}<1$, which may indicate that neither additive nor synergistic effects between LYC and ATOC towards the scavenging of the ABTS radical cation took place in them. These interactions can be considered as antagonistic.

The highest SE value was detected for M15, this mixture presenting the highest content of ATOC. Overall, it was observed that the SE values increased as the ATOC levels did and that in all the cases where possible synergistic effects were observed the amount of ATOC was at least 2 times higher than that of LYC. However, interestingly, the LYC/ATOC ratio could not explain the trends in the $\mathrm{SE}$ values. A self-explanatory example of this is that such ratio was the same (0.5) in both M1 and M26, albeit they had markedly different SE values (0.66 and 1.02, Table 3). Taken together, these results point to the fact that the interactions between LYC and ATOC towards the scavenging of the $\mathrm{ABTS}^{+}$are difficult to explain and cannot be readily predicted only from their relative levels.

Overall, these results were in accordance with those reported elsewhere. Zanfini et al. (2010) evaluated the synergistic interactions in LYC, ATOC, $\beta$-carotene and lutein mixtures in terms of TEAC. They reported that the highest synergistic effects were detected for ATOC-LYC mixtures and that the one that had higher content of ATOC showed highest synergistic effect $(>\mathrm{SE})$. Others researchers reported that certain combinations of lycopene with other compounds showed an improved AC towards DPPH (Liu et al., 2008). On the other hand, there are also studies that suggest that the TEAC of a mixture of hydrophilic and lipophilic antioxidants can be additive (van den Berg, Haenen, van den Berg, \& Bast, 1999).

Based on these results, it can be concluded that the lycopene and $\alpha$-tocopherol present in a mixture can interact, and their interactions can affect the total antioxidant capacity of a solution, in this case extracts from tomato samples. These evidences suggest that the study of the existence of interactions between antioxidants should be fostered to generate new valuable knowledge for the industry with a view to develop more optimized food ingredients.

\subsection{Reflectance measurements}

As explained before, lycopene seemed to be the antioxidant with the highest contribution to the TEAC of the extracts. LYC is the main contributor to the colour of tomato products, which is an important quality attribute much related to their acceptability. Since tomato colour is usually controlled in the industry by means of reflectance measurements, we hypothesized that not only the LYC levels but also the TEAC could be rapidly assessed from reflectance values at certain wavelengths. This can offer advantages for the quality control of tomato products in the industry due to the inherent advantages of such measurements.

Table 2

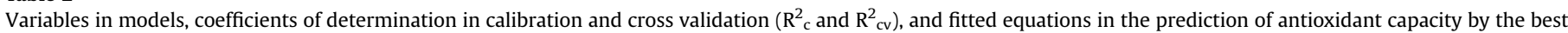
subsets multiple linear regressions based on lycopene isomers, phytoene, phytofluene and $\alpha$-tocopherol content (in $\mathrm{g} / \mathrm{kg} \mathrm{DW}$ ).

\begin{tabular}{|c|c|c|c|c|}
\hline Model & Variables & Equations & $\mathrm{R}^{2}{ }_{\mathrm{c}}$ & $\mathrm{R}_{\mathrm{cv}}^{2}$ \\
\hline 1 & all-E-LYC, $\sum Z$-LYC & $\mathrm{AC}=3.36($ all-E $-\mathrm{LYC})+11.31\left(\sum \mathrm{Z}-\mathrm{LYC}\right)+0.91$ & 0.868 & 0.842 \\
\hline 2 & $\begin{array}{l}\text { 15Z-LYC, 13Z-LYC, 9Z-LYC, } \\
\text { all-E-LYC, 5Z-LYC }\end{array}$ & $\begin{array}{l}\mathrm{AC}=-9.05(15 Z-\mathrm{LYC})+58.24(13 \mathrm{Z}-\mathrm{LYC})-88.84(9 \mathrm{Z}-\mathrm{LYC})+ \\
3.24(\text { all- } E-\mathrm{LYC})+3.67(5 Z-\mathrm{LYC})+1.34\end{array}$ & 0.895 & 0.856 \\
\hline 3 & ATOC, $\sum$ LYC & $\mathrm{AC}=0.98(\mathrm{ATOC})+3.74\left(\sum \mathrm{LYC}\right)+0.96$ & 0.870 & 0.846 \\
\hline 4 & $\begin{array}{l}\text { ATOC, 15Z-LYC, 13Z-LYC, } \\
\text { 9Z-LYC, all-E-LYC, 5Z-LYC }\end{array}$ & $\begin{array}{l}\mathrm{AC}=0.52(\mathrm{ATOC})-4.94(15 Z-\mathrm{LYC})+54.64(13 Z-\mathrm{LYC})- \\
96.14(9 \mathrm{Z}-\mathrm{LYC})+3.16(\text { all }-E-\mathrm{LYC})+3.43(5 Z-\mathrm{LYC})+1.31\end{array}$ & 0.896 & 0.846 \\
\hline 5 & ATOC, PT, PF, $\sum$ LYC & $\mathrm{AC}=-0.47(\mathrm{ATOC})-0.18(\mathrm{PT})+19.36(\mathrm{PF})+3.42\left(\sum \mathrm{LYC}\right)+1.15$ & 0.877 & 0.842 \\
\hline 6 & $\begin{array}{l}\text { ATOC, PT, PF, 15Z-LYC, } \\
\text { 13Z-LYC. 9Z-LYC, all-E-LYC, 5Z-LYC }\end{array}$ & $\begin{array}{l}\mathrm{AC}=-0.97(\mathrm{ATOC})-0.67(\mathrm{PT})+24.73(\mathrm{PF})+5.43(15 \mathrm{Z}-\mathrm{LYC})+ \\
55.93(13 \mathrm{Z}-\mathrm{LYC})-82.28(9 \mathrm{Z}-\mathrm{LYC})+2.81(\text { all-E }-\mathrm{LYC})+3.45(5 \mathrm{Z}-\mathrm{LYC})+1.47\end{array}$ & 0.902 & 0.842 \\
\hline
\end{tabular}

Abbreviations: LYC, lycopene: PT, phytoene; PF, phytofluene; ATOC, $\alpha$-tocopheroL; AC, antioxidant capacity. 
Table 3

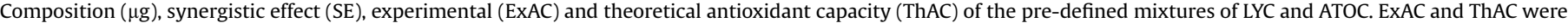
calculated considering the absorbance drop $(\triangle \mathrm{Abs})$ of the ABTS radical cation solution.

\begin{tabular}{|c|c|c|c|c|c|c|c|}
\hline Mixtures & LYC $(\mu \mathrm{g})$ & ATOC $(\mu \mathrm{g})$ & LYC/ATOC & $\mathrm{ExAC}^{\mathrm{a}}$ & ThAC & $\mathrm{SE}^{\mathrm{b}}$ & $p$-values ${ }^{c}$ \\
\hline 1 & 0.324 & 0.660 & 0.5 & $0.114 \pm 0.010$ & 0.173 & 0.66 & $<0.001$ \\
\hline 2 & 0.324 & 1.319 & 0.2 & $0.182 \pm 0.008$ & 0.246 & 0.74 & $<0.001$ \\
\hline 3 & 0.324 & 2.638 & 0.1 & $0.318 \pm 0.016$ & 0.330 & 0.96 & 0.144 \\
\hline 4 & 0.324 & 3.957 & 0.1 & $0.433 \pm 0.013$ & 0.427 & 1.01 & 0.320 \\
\hline 5 & 0.324 & 5.276 & 0.1 & $0.586 \pm 0.018$ & 0.553 & 1.06 & 0.003 \\
\hline 6 & 0.594 & 0.958 & 0.6 & $0.185 \pm 0.007$ & 0.219 & 0.84 & $<0.001$ \\
\hline 7 & 0.594 & 1.916 & 0.3 & $0.256 \pm 0.007$ & 0.295 & 0.87 & $<0.001$ \\
\hline 8 & 0.594 & 2.873 & 0.2 & $0.367 \pm 0.016$ & 0.374 & 0.98 & 0.355 \\
\hline 9 & 0.594 & 3.831 & 0.2 & $0.423 \pm 0.013$ & 0.464 & 0.91 & $<0.001$ \\
\hline 10 & 0.594 & 5.747 & 0.1 & $0.540 \pm 0.018$ & 0.605 & 0.89 & $<0.001$ \\
\hline 11 & 0.648 & 0.660 & 1.0 & $0.180 \pm 0.016$ & 0.226 & 0.79 & $<0.001$ \\
\hline 12 & 0.648 & 1.319 & 0.5 & $0.246 \pm 0.008$ & 0.299 & 0.83 & $<0.001$ \\
\hline 13 & 0.648 & 2.638 & 0.2 & $0.372 \pm 0.012$ & 0.383 & 0.97 & 0.139 \\
\hline 14 & 0.648 & 3.957 & 0.2 & $0.505 \pm 0.032$ & 0.480 & 1.05 & 0.109 \\
\hline 15 & 0.648 & 5.276 & 0.1 & $0.659 \pm 0.008$ & 0.605 & 1.09 & $<0.001$ \\
\hline 16 & 0.971 & 0.660 & 1.5 & $0.235 \pm 0.018$ & 0.276 & 0.85 & 0.001 \\
\hline 17 & 0.971 & 1.319 & 0.7 & $0.300 \pm 0.010$ & 0.349 & 0.86 & $<0.001$ \\
\hline 18 & 0.971 & 2.638 & 0.4 & $0.439 \pm 0.015$ & 0.433 & 1.01 & 0.357 \\
\hline 19 & 0.971 & 3.957 & 0.2 & $0.536 \pm 0.011$ & 0.530 & 1.01 & 0.235 \\
\hline 20 & 1.188 & 0.958 & 1.2 & $0.286 \pm 0.007$ & 0.310 & 0.92 & $<0.001$ \\
\hline 21 & 1.188 & 1.916 & 0.6 & $0.347 \pm 0.010$ & 0.385 & 0.90 & $<0.001$ \\
\hline 22 & 1.188 & 2.873 & 0.4 & $0.435 \pm 0.014$ & 0.464 & 0.94 & $<0.001$ \\
\hline 23 & 1.188 & 3.831 & 0.3 & $0.529 \pm 0.021$ & 0.554 & 0.95 & 0.010 \\
\hline 24 & 1.295 & 0.660 & 2.0 & $0.273 \pm 0.011$ & 0.322 & 0.85 & $<0.001$ \\
\hline 25 & 1.295 & 1.319 & 1.0 & $0.362 \pm 0.017$ & 0.395 & 0.92 & 0.003 \\
\hline 26 & 1.295 & 2.638 & 0.5 & $0.491 \pm 0.011$ & 0.479 & 1.02 & 0.038 \\
\hline 27 & 1.619 & 0.660 & 2.5 & $0.325 \pm 0.026$ & 0.367 & 0.89 & 0.007 \\
\hline 28 & 1.619 & 1.319 & 1.2 & $0.432 \pm 0.015$ & 0.439 & 0.98 & 0.409 \\
\hline 29 & 1.782 & 0.958 & 1.9 & $0.362 \pm 0.018$ & 0.392 & 0.92 & 0.189 \\
\hline 30 & 1.782 & 1.916 & 0.9 & $0.435 \pm 0.015$ & 0.467 & 0.93 & 0.133 \\
\hline 31 & 1.782 & 2.873 & 0.6 & $0.527 \pm 0.004$ & 0.547 & 0.96 & 0.905 \\
\hline 32 & 1.782 & 3.831 & 0.5 & $0.557 \pm 0.004$ & 0.637 & 0.87 & 0.270 \\
\hline 33 & 2.376 & 0.958 & 2.5 & $0.451 \pm 0.018$ & 0.482 & 0.94 & 0.280 \\
\hline 34 & 2.376 & 1.916 & 1.2 & $0.509 \pm 0.012$ & 0.557 & 0.91 & 0.374 \\
\hline 35 & 2.376 & 2.873 & 0.8 & $0.579 \pm 0.017$ & 0.637 & 0.91 & $<0.001$ \\
\hline
\end{tabular}

Abbreviations: LYC, lycopene; ATOC, $\alpha$-tocopherol; ThAC, theoretical antioxidant capacity value; ExAC, experimental antioxidant capacity value; SE, synergistic effects.

a ExAC values are reported as mean $(n=5) \pm$ S.D.

b The existence of synergistic effect was considered when $\mathrm{SE}>1$.

c Bold values are significantly different.

Given that the instrumental reflectance readings were taken at $2 \mathrm{~nm}$ intervals and that, therefore, ca. 200 values were obtained for each sample, it was necessary to simplify the number of reflectance data. For this purpose, for this part of the study, they were arbitrarily considered at $8 \mathrm{~nm}$ intervals. Afterwards, SLDA was carried out to determine the most appropriate wavelengths to discriminate between fresh and processed tomatoes.

Considering the reflectances at different wavelengths, forward stepwise analysis was applied and 36 variables were chosen. The mathematical model selected 23 variables having the greatest discriminatory power with a classification rate of $100 \%$ in all cases. In the graphical representation of the samples within the discriminant space (see Supplementary material Fig. 1), it can be observed that the variables used lead to the division of the samples into two clearly distinguishable groups.

Considering the wavelengths selected by the model, a forward stepwise multiple regression procedure was applied to obtain equations that allowed to estimate the total LYC content or the TEAC from reflectance readings (Table 4). Statistically significant regression coefficients were obtained in all cases. This indicated that the values obtained from the HPLC analyses and the ABTS ${ }^{+}$ assays were significantly correlated to those predicted from the reflectance readings at the selected wavelengths. To test whether the values obtained by both methodologies were significantly different or not, the actual experimental values were compared to those computed from the equations (Fig. 1) by means of ANOVA. The $p$ values obtained ranged from 0.886 to 0.999 , which indicated that there were no significant differences $(p<0.05)$ (Table 4$)$.

These results indicated that it is feasible to attain a good estimation of the lycopene content or TEAC in both types of tomato samples from reflectance readings. Due to the inherent advantages of the instrumental measurements of the visible reflection spectra (simplicity, rapidity, versatility, portability, etc.) this novel approach can be applied for the rapid assessment of the in vitro AC of carotenoid-containing foods in the field, the industry or even the market.

\section{Conclusion}

The levels of LYC, PT, PF and ATOC were considerably higher in the processed tomato products analysed (approximately, 2.7-fold, 2.6-fold, 3.4-fold and 3.4-fold, respectively). The average TEAC value of these samples was ca. 2.4-fold higher than that of the fresh samples.

Good simple correlations were found between the TEAC values and the LYC and ATOC levels, as it could be expected beforehand. More importantly, statistically significant good correlations were also found for PT and PF, two little studied carotenoids present in high amounts in tomatoes. The possible AC of these colourless carotenoids have been paid little attention to date and deserves further evaluation. Indeed, in our experiments PF exhibited a higher TEAC than ATOC, which was unexpected and noteworthy. The theoretical individual contribution of all the compounds studied in the TEAC was evaluated by multiple regression analysis. 
Table 4

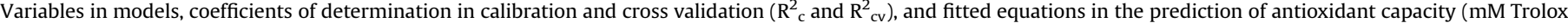
equivalents/kg DW) and lycopene content ( $\sum$ LYC) (in $\mathrm{g} / \mathrm{kg} \mathrm{DW}$ ) by the best subsets multiple linear regressions based on reflectance measurement.

\begin{tabular}{|c|c|c|c|c|c|c|}
\hline Model & & Variables (nm) & Equat & & $\mathrm{R}_{\mathrm{c}}^{2}$ & $\mathrm{R}_{\mathrm{cv}}^{2}$ \\
\hline Fresh & $\mathrm{AC}$ & $\left(R_{550}\right),\left(R_{694}\right),\left(R_{582}\right),\left(R_{390}\right)$ & Eq. 1 & $\begin{array}{l}A C=14.63\left(R_{550}\right)+10.80\left(R_{694}\right)-28.77 \\
\left(R_{582}\right)-7.33\left(R_{390}\right)+3.93\end{array}$ & 0.892 & 0.814 \\
\hline Fresh & LYC & $\left(R_{574}\right),\left(R_{646}\right),\left(R_{606}\right),\left(R_{390}\right)$ & Eq. 2 & $\begin{array}{l}\sum L Y C=-0.25\left(R_{574}\right)+3.65\left(R_{646}\right)-5.42 \\
\left(R_{606}\right)-1.86\left(R_{390}\right)+1.38\end{array}$ & 0.900 & 0.794 \\
\hline Processed & $\mathrm{AC}$ & $\begin{array}{l}\left(R_{398}\right),\left(R_{686}\right),\left(R_{390}\right),\left(R_{622}\right), \\
\left(R_{734}\right),\left(R_{646}\right),\left(R_{710}\right)\end{array}$ & Eq. 3 & $\begin{array}{l}A C=-150.30\left(R_{398}\right)-3.94\left(R_{686}\right)+90.81 \\
\left(R_{390}\right)-68.34\left(R_{622}\right)-19.03\left(R_{734}\right)+60.37 \\
\left(R_{646}\right)+43.24\left(R_{710}\right)-2.95\end{array}$ & 0.724 & 0.377 \\
\hline Processed & LYC & $\left(R_{694}\right),\left(R_{622}\right),\left(R_{630}\right),\left(R_{654}\right)$ & Eq. 4 & $\begin{array}{l}\sum L Y C=19.85\left(R_{694}\right)-30.32\left(R_{622}\right)+34.84 \\
\left(R_{630}\right)-21.63\left(R_{654}\right)-2.17\end{array}$ & 0.495 & 0.245 \\
\hline All & $A C$ & $\begin{array}{l}\left(R_{590}\right),\left(R_{694}\right),\left(R_{734}\right),\left(R_{654}\right), \\
\left(R_{710}\right),\left(R_{614}\right),\left(R_{622}\right),\left(R_{606}\right)\end{array}$ & Eq. 5 & $\begin{array}{l}A C=-4.23\left(R_{590}\right)+24.58\left(R_{694}\right)-23.08 \\
\left(R_{734}\right)-17.28\left(R_{654}\right)+29.16\left(R_{710}\right)-170.24 \\
\left(R_{614}\right)+114.95\left(R_{622}\right)+50.38\left(R_{606}\right)-1.89\end{array}$ & 0.852 & 0.790 \\
\hline All & LYC & $\begin{array}{l}\left(R_{590}\right),\left(R_{646}\right),\left(R_{614}\right),\left(R_{654}\right), \\
\left(R_{710}\right),\left(R_{558}\right),\left(R_{734}\right),\left(R_{694}\right)\end{array}$ & Eq. 6 & $\begin{array}{l}\sum L Y C=8.56\left(R_{590}\right)+14.94\left(R_{646}\right)-15.17 \\
\left(R_{614}\right)-8.99\left(R_{654}\right)+5.81\left(R_{710}\right)-5.70\left(R_{558}\right)-2.12 \\
\left(R_{734}\right)+3.91\left(R_{694}\right)-1.22\end{array}$ & 0.839 & 0.745 \\
\hline
\end{tabular}

Abbreviations: LYC, lycopene: AC, antioxidant capacity.
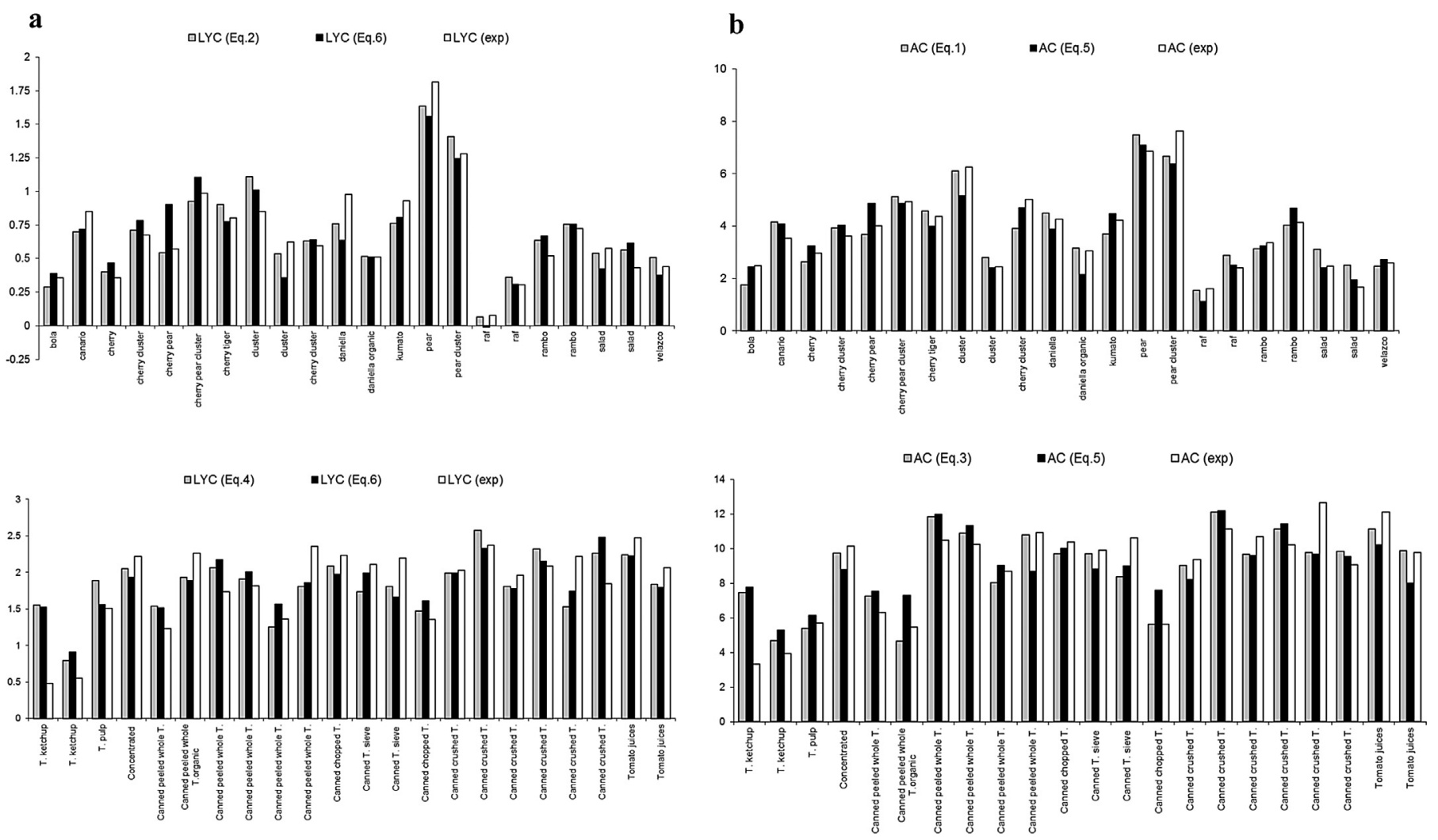

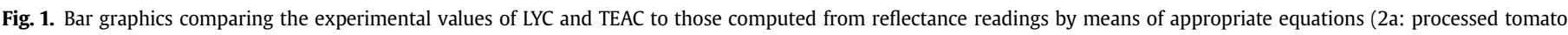
samples; $2 \mathrm{~b}$ : tomato fruits). No significant differences between values were observed $(p<0.05)$.

Considering the beta coefficients, it seems that LYC is the compound with the highest impact on the AC, followed by PF.

Given that both LYC and ATOC are used as food ingredients and exhibit AC it appeared interesting to assess whether there can be interactions between them in terms of the scavenging of the ABTS $^{+}$. The results of the experiments conducted indicated that the decreases in the absorbance of the $\mathrm{ABTS}^{+}$solutions owed to pre-defined LYC and ATOC amounts were not additive. Rather, interactions seem to take place, some of them being synergistic. Nonetheless, the LYC/ATOC ratio cannot be used on its own to predict the kind of interaction that occurs.

Lastly, it has been shown that the LYC levels and TEAC values can be rapidly estimated from reflectance measurements by considering the readings at certain wavelengths and applying appropriate equations. The values so computed were significantly correlated with the values obtained by HPLC and the TEAC assay. More importantly, it was noticed that there were no significant differences $(p<0.05)$ between the values calculated from the equations and the experimental data.

More research is needed to clarify the role of lycopene, tomato carotenoids, and their metabolites in producing the beneficial effects associated with their intake, since the antioxidant capacity of foods based on their capability of scavenging free radicals in vitro refer to a property of the foods, and it is not established that this 
capability exerts a beneficial physiological effect in humans (EFSA, 2011).

\section{Acknowledgements}

AJMM acknowledges funding from the European Union (Marie Curie Reintegration Grant ERG-224789, INTERCAROTEN) and from the Spanish Secretaría de Estado de Investigación, Desarrollo e Innovación (Ministerio de Economía y Competitividad, project ref. AGL2012-37610, co-funded by FEDER). The authors are members of the IBERCAROT (http://carotenoides.us.es) network, funded by CYTED (ref. 112RT0445).

\section{Appendix A. Supplementary data}

Supplementary data related to this article can be found at http:// dx.doi.org/10.1016/j.lwt.2015.08.068.

\section{References}

Abushita, A. A., Daood, H. G., \& Biacs, P. A. (2000). Change in arotenoids and antioxidant vitamins in tomato as a function of varietal and technological factors. Journal of Agricultural and Food Chemistry, 48, 2075-2081.

Adalid, A. M., Roselló, S., \& Nuez, F. (2010). Evaluation and selection of tomato accessions (Solanum section Lycopersicon) for content of lycopene, $\beta$-carotene and ascorbic acid. Journal of Food Composition and Analysis, 23, 613-618.

van den Berg, R., Haenen, G. R. M. M., van den Berg, H., \& Bast, A. (1999). Applicability of an improved Trolox equivalent antioxidant capacity (TEAC) assay for evaluation of antioxidant capacity measurements of mixtures. Food Chemistry, $66,511-517$

Borguini, R. G., \& Ferraz Da Silva Torres, E. (2009). Tomatoes and tomato products as dietary sources of antioxidants. Food Reviews International, 25, 313-325.

Dewanto, V., Wu, X., Adom, K. K., \& Liu, R. H. (2002). Thermal processing enhances the nutritional value of tomatoes by increasing total antioxidant activity. Journal of Agricultural and Food Chemistry, 50, 3010-3014.

EFSA. (2011). Guidance on the scientific requirements for health claims related to antioxidants, oxidative damage and cardiovascular health. EFSA Journal, 9, 2474

Erba, D., Casiraghi, M. C., Ribas-Agustí, A., Cáceres, R., Marfá, O., \& Castellari, M. (2013). Nutritional value of tomatoes (Solanum lycopersicum L.) grown in greenhouse by different agronomic techniques. Journal of Food Composition and Analysis, 31, 245-251.

Fröhlich, K., Conrad, J., Schmid, A., Breithaupt, D. E., \& Böhm, V. (2007). Isolation and structural elucidation of different geometrical isomers of lycopene. International Journal for Vitamin and Nutrition Research, 77, 369-375.

Fröhlich, K., Kaufmann, K., Bitsch, R., \& Böhm, V. (2006). Effects of ingestion of tomatoes, tomato juice and tomato purée on contents of lycopene isomers, tocopherols and ascorbic acid in human plasma as well as on lycopene isomer pattern. British Journal of Nutrition, 95, 734-741.

Fuhrman, B., Volkova, N., Rosenblat, M., \& Aviram, M. (2000). Lycopene synergistically inhibits LDL oxidation in combination with vitamin E, glabridin, rosmarinic acid, carnosic acid, or garlic. Antioxidants \& Redox Signaling, 2, 491-506.

Galano, A., \& Francisco-Marquez, M. (2009). Reactions of OOH radical with $\beta$ carotene, lycopene, and torulene: hydrogen atom transfer and adduct formation mechanisms. The Journal of Physical Chemistry B, 113, 11338-11345.

Giovannucci, E. (2005). Tomato products, lycopene, and prostate Cancer: a review of the epidemiological literature. The Journal of Nutrition, 135, 2030S-2031S.

Guo, W. H., Tu, C. Y., \& Hu, C. H. (2008). Cis/Trans isomerizations of $\beta$-carotene and lycopene: a theoretical study. The Journal of Physical Chemistry B, 112, $12158-12167$.
Heredia, A., Peinado, I., Barrera, C., \& Grau, A. A. (2009). Influence of process variables on colour changes, carotenoids retention and cellular tissue alteration of cherry tomato during osmotic dehydration. Journal of Food Composition and Analysis, 22, 285-294.

Honest, K. N., Zhang, H. W., \& Zhang, L. (2011). Lycopene: isomerization effects on bioavailability and bioactivity properties. Food Reviews International, 27 $248-258$.

Kun, Y., Ssonko Lule, U., \& Xiao-Lin, D. (2006). Lycopene: its properties and relationship to human health. Food Reviews International, 22, 309-333.

Liu, D., Shi, J., Colina Ibarra, A., Kakuda, Y., \& Jun Xue, S. (2008). The scavenging capacity and synergistic effects of lycopene, vitamin E, vitamin C, and [beta]carotene mixtures on the DPPH free radical. LWT - Food Science and Technology, 41, 1344-1349.

Martínez, A., Stinco, C. M., \& Meléndez-Martínez, A. J. (2014). Free radical scavenging properties of phytofluene and phytoene isomers as compared to lycopene: a combined experimental and theoretical study. The Journal of Physical Chemistry B, 118, 9819-9825.

Meléndez-Martínez, A. J., Fraser, P. D., \& Bramley, P. M. (2010). Accumulation of health promoting phytochemicals in wild relatives of tomato and their contribution to in vitro antioxidant activity. Phytochemistry, 71, 1104-1114.

Meléndez-Martínez, A. J., Mapelli-Brahm, P., Benítez-González, A., \& Stinco, C. M. (2015). A comprehensive review on the colorless carotenoids phytoene and phytofluene. Archives of Biochemistry and Biophysics, 572, 188-200.

Meléndez-Martínez, A. J., Nascimento, A. F., Wang, Y., Liu, C., Mao, Y., \& Wang, X. D. (2013b). Effect of tomato extract supplementation against high-fat diet-induced hepatic lesions. Hepatobiliary Surgery and Nutrition, 2, 198-208.

Meléndez-Martínez, A. J., Stinco, C. M., Liu, C. \& Wang, X. D. (2013a). A simple HPLC method for the comprehensive analysis of cis/trans (Z/E) geometrical isomers of carotenoids for nutritional studies. Food Chemistry, 138, 1341-1350.

Miller, N. J., Sampson, J., Candeias, L. P., Bramley, P. M., \& Rice-Evans, C. A. (1996) Antioxidant activities of carotenes and xanthophylls. FEBS Letters, 384 $240-242$.

Raffo, A., Leonardi, C., Fogliano, V., Ambrosino, P., Salucci, M., Gennaro, L., et al (2002). Nutritional value of cherry tomatoes (Lycopersicon esculentum cv. Naomi F1) harvested at different ripening stages. Journal of Agricultural and Food Chemistry, 50, 6550-6556.

Re, R., Pellegrini, N., Proteggente, A., Pannala, A., Yang, M., \& Rice-Evans, C. (1999). Antioxidant activity applying an improved ABTS radical cation decolorization assay. Free Radical Biology \& Medicine, 26, 1231-1237.

Shi, J., Qu, Q., Kakuda, Y., Xue, S. J., Jiang, Y., Koide, S., et al. (2007). Investigation of the antioxidant and synergistic activity of lycopene and other natural antioxidants using LAME and AMVN model systems. Journal of Food Composition and Analysis, 20, 603-608.

Shixian, O., Dai, Y., Kakuda, Y., Shi, J., Mittal, G., Yeung, D., et al. (2005). Synergistic anti-oxidative effects of lycopene with other bioactive compounds. Food Reviews International, 21, 295-311.

Stahl, W., \& Sies, H. (1992). Uptake of lycopene and its geometrical isomers is greater from heat-processed than from unprocessed tomato juice in humans. The Journal of Nutrition, 122, 2161-2166.

Stinco, C. M., Rodríguez-Pulido, F. J., Escudero-Gilete, M. L., Gordillo, B., Vicario, I. M. \& Meléndez-Martínez, A. J. (2013). Lycopene isomers in fresh and processed tomato products: correlations with instrumental color measurements by digita image analysis and spectroradiometry. Food Research International, 50, 111-120.

Tedeschi, P., Coïsson, J. D., Maietti, A., Cereti, E., Stagno, C., Travaglia, F., et al. (2011) Chemotype and genotype combined analysis applied to tomato (Lycopersicon esculentum Mill.) analytical traceability. Journal of Food Composition and Analysis, 24, 131-139.

Thakur, B. R., Singh, R. K., \& Nelson, P. E. (1996). Quality attributes of processed tomato products: a review. Food Reviews International, 12, 375-401.

Woodall, A. A., Lee, S. W.-M., Weesie, R. J., Jackson, M. J., \& Britton, G. (1997) Oxidation of carotenoids by free radicals: relationship between structure and reactivity. Biochimica et Biophysica Acta (BBA) - General Subjects, 1336, 33-42.

Zanfini, A., Corbini, G., La Rosa, C., \& Dreassi, E. (2010). Antioxidant activity of tomato lipophilic extracts and interactions between carotenoids and [alpha]tocopherol in synthetic mixtures. LWT - Food Science and Technology, 43, 67-72. 